\title{
Press that button again, please
}

$\mathrm{T}$ hey're called brain pacemakers and if scientists can just sort out the tricky task of determining where exactly to place the electrical wiring in all the white and grey matter found in the human brain, they may have the potential to make obsolete the use of drugs and other therapies in the treatment of depression and other mental disorders.

The notion of sending electrical impulses into the brain to cure disease isn't entirely novel; it's being used to treat chronic pain and Parkinson Disease. But deep brain stimulation (DBS), in the parlance of the psychiatric surgery community, as a therapy for mental illness such as treatment-resistant depression is a much more recent development, and one which researchers say needs more study.

It involves implanting a thin, somewhat flexible wire into a certain region of the brain - in the case of depression, the subcallosal cingulate. The wire has four contacts, each of which can serve as points of stimulation, says Dr. Paul Holtzheimer, associate professor of psychiatry and surgery at Dartmouth Medical School and director of Mood Disorders Service at Dartmouth Hitchcock Medical Center in New Hampshire.

"That wire, as it comes out of the skull, is then connected to extension wires that are tunnelled under the skin and typically down the side of the neck, over the collarbone and down into the chest wall area. And that's connected to a computer battery pack that controls the stimulation," he says. The entire system is implanted in the patient's body. Its battery pack must be replaced every 18 to 24 months.

DBS appears to alter the way regions of the brain communicate with one another, Holtzheimer says. It may be that the device is "establishing a more normal rhythm within the brain ... and not allowing that change back into what would be a depressive rhythm."

Holtzheimer (then at the Emory University School of Medicine in Atlanta,

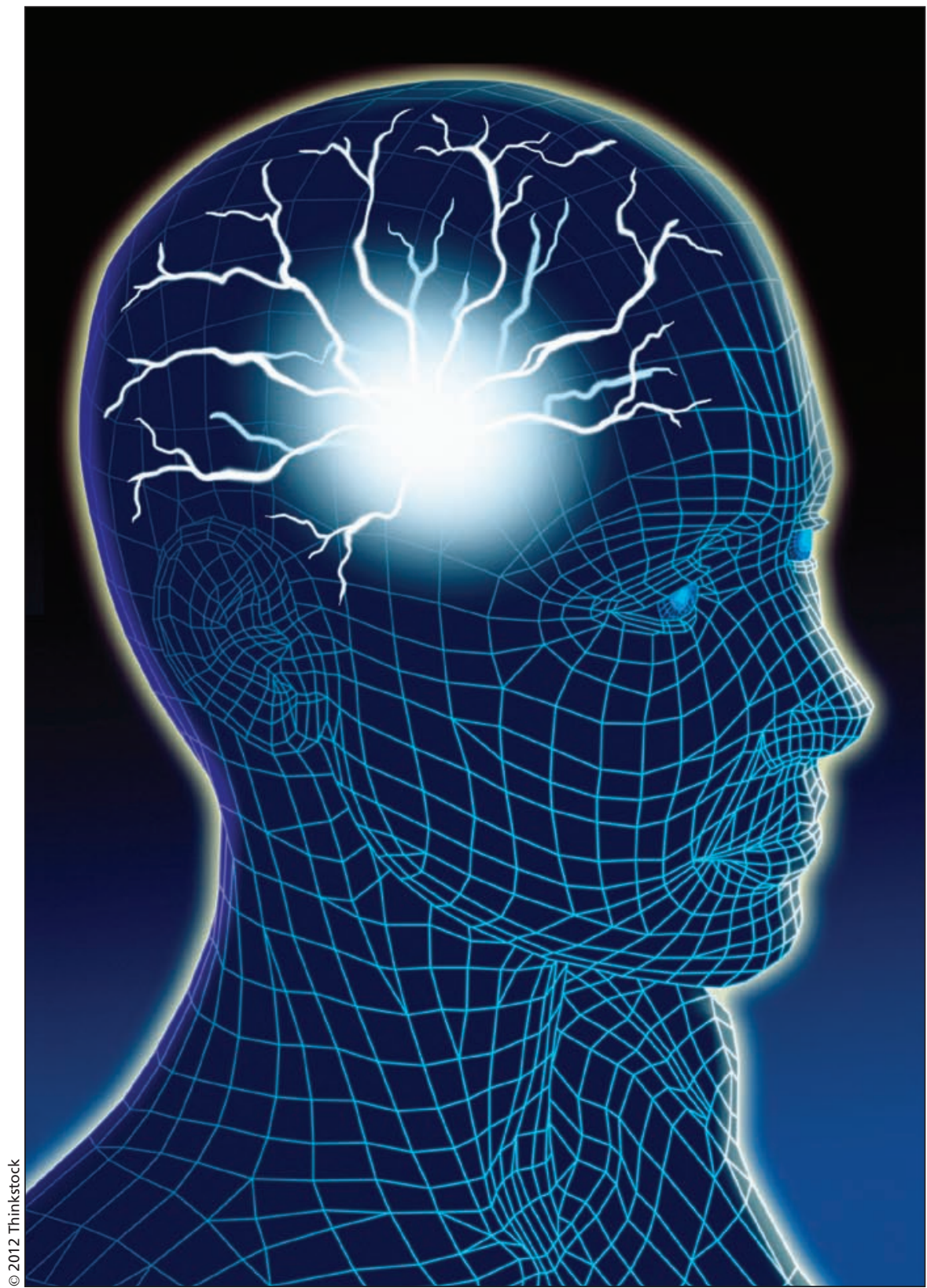

Deep brain stimulation appears to alter the way regions of the brain communicate with one another.

Georgia) and colleagues implanted brain pacemakers in 10 patients with major depressive disorder (MDD) and seven with bipolar II (BP) disorder and found that "a significant decrease in depression and increase in function were associated with chronic stimulation. Remission and response were seen in 3 patients $(18 \%)$ and $7(41 \%)$ after 24 weeks $(n=17), 5(36 \%)$ and 5 $(36 \%)$ after 1 year $(n=14)$, and 7 $(58 \%)$ and $11(92 \%)$ after 2 years $(n=$ 2 ) of active stimulation. No patient achieving remission experienced a spontaneous relapse. Efficacy was similar for patients with MDD and those 
with BP. Chronic DBS was safe and well tolerated, and no hypomanic or manic episodes occurred. A modest sham stimulation effect was found, likely due to a decrease in depression after the surgical intervention but prior to entering the sham phase" (Arch Gen Psychiatry 2012;69:150-8).

Holtzheimer's group define patients with treatment-resistant depression as those "with a moderate-to-severe major depressive episode after at least 4 adequate antidepressant treatments" such as cognitive behavioural or electroconvulsive therapy.

At least one patient involved in the study, who asks that she be identified only as Melanie, says "my whole world is different now. It changed everything."

Diagnosed with depression at the age of 20, she has been in treatment for over 14 years, receiving various combinations of medications and electroconvulsive therapy. "Nothing seemed to work. I just sort of survived but that was about all I was doing," she says.

A brain pacemaker was implanted in her in February 2010 and repositioned in August 2010. Melanie felt the effects immediately after doctors tested the device after her August surgery. "I often felt like I needed to claw something out of [my head, particularly, a persistent noise]. ... You know, sort of like nails on a chalkboard and it makes you cringe or recoil from it. I just always had that feeling in my head, like I was in recoil and it was what I sensed when they turned it off."

"It was about turning down a volume, like something that was overactive. I couldn't access the parts of my brain that I needed to make normal decisions or live a normal life because everything was on par with screaming all the time."

Melanie adds that the pacemaker has allowed her to return to normal sleep patterns, and to hold down a job, something she couldn't have imagined two years ago. But she stresses that not all her problems have been solved. "It wasn't an instant happy fix. It sort of took the obstacle out of the way so that I could do the work."

Holzheimer stresses that more clinical trials are needed before brain pacemakers are implanted willy-nilly among those with treatment-resistant depression. While no adverse effects have been evident in 17 patients, that may not be true once a greater number of people undergo clinical trials. "It's going to take more time and more patients to truly know what the possible range of side effects can be" other than those typically associated with brain surgery, such as hemorrhage or infection.

There may also be emotional risks for patients who are implanted with brain pacemakers, says Dr. Jeff Daskalakis, director of the Brain Stimulation Treatment and Research Program at the Centre for Addiction and Mental Health in Toronto, Ontario, and associate professor of psychiatry at the University of Toronto. "If patients don't respond, they really do feel like they're at the end of their rope."

DBS is really a last choice because of its invasive nature, Daskalakis says. Doctors are "putting probes into people's brains which would make people nervous about getting the treatment until they are sure they've failed all the medications" and explored all other alternatives.

But it may be an answer for those for whom more traditional therapies have failed and who "are now more and more commonly turning to alternative forms of treatment" such as brain pacemakers, Daskalakis says. - Erin Walkinshaw, Mississauga, Ont.

CMAJ 2012. DOI:10.1503/cmaj.109-4112 